\title{
Large-Eddy Simulation of Very-Large-Scale Motions in the Neutrally Stratified Atmospheric Boundary Layer
}

\author{
Jiannong Fang · Fernando Porté-Agel
}

Received: 21 August 2014 / Accepted: 22 January 2015 / Published online: 20 February 2015

C) Springer Science+Business Media Dordrecht 2015

\begin{abstract}
Large-eddy simulation is used to investigate very-large-scale motions (VLSMs) in the neutrally stratified atmospheric boundary layer at a very high friction Reynolds number, $R e_{\tau} \sim \mathcal{O}\left(10^{8}\right)$. The vertical height of the computational domain is $L_{z}=1000 \mathrm{~m}$, which corresponds to the thickness of the boundary layer. In order to make sure that the largest flow structures are properly resolved, the horizontal domain size is chosen to be $L_{x}=32 \pi L_{z}$ and $L_{y}=4 \pi L_{z}$, which is much larger than the standard domain size, especially in the streamwise direction (i.e., the direction of elongation of the flow structures). It is shown that the contributions to the resolved turbulent kinetic energy and the resolved shear stress from streamwise wavelengths larger than $10 L_{z}$ are up to 27 and $31 \%$ respectively. Therefore, the large computational domain adopted here is essential for the purpose of investigating VLSMs. The spatially coherent structures associated with VLSMs are characterized through flow visualization and statistical analysis. The instantaneous velocity fields in horizontal planes give evidence of streamwise-elongated flow structures of low-speed fluid with negative fluctuation of the streamwise velocity component, and which are flanked on either side by similarly elongated high-speed structures. The pre-multiplied power spectra and two-point correlations indicate that the scales of these streak-like structures are very large, up to $20 L_{z}$ in the streamwise direction and $0.6 L_{z}$ in the spanwise direction. These features are similar to those found in the logarithmic and outer regions of laboratory-scale boundary layers by direct numerical simulation and experiments conducted at low to moderate Reynolds numbers. The three-dimensional correlation map and conditional average of the three components of velocity further indicate that the low-speed and high-speed regions possess the same elongated ellipsoid-like structure, which is inclined upward along the streamwise direction, and they are accompanied by counter-rotating roll modes in the cross-section perpendicular
\end{abstract}

J. Fang · F. Porté-Agel ( $\square)$

Wind Engineering and Renewable Energy Laboratory (WIRE), Institute of Environmental Engineering (IIE), School of Architecture, Civil and Environmental Engineering (ENAC), École Polytechnique Fédérale de Lausanne (EPFL), 1015 Lausanne, Switzerland e-mail: fernando.porte-agel@epfl.ch

J. Fang

e-mail: jiannong.fang@epfl.ch 
to the streamwise direction. These results are in agreement with recent observations in the atmospheric surface layer.

Keywords Large-eddy simulation - Turbulent boundary layer - Coherent structures . Very-large-scale motions

\section{Introduction}

Recent experimental studies of turbulent boundary layers (Tomkins and Adrian 2003; Ganapathisubramani et al. 2003; Hambleton et al. 2006; Balakumar and Adrian 2007; Hutchins and Marusic 2007a; Mathis et al. 2009; Dennis and Nickels 2011; Tang et al. 2012) have revealed the existence of very-large-scale structures in the logarithmic and outer regions, with sizes in the range $10 \delta-20 \delta$, where $\delta$ is the boundary-layer thickness. Typical features of these structures include peaks occurring at low frequencies in the pre-multiplied energy spectra and highly streamwise-elongated, alternating low- and high-speed, meandering zones in the instantaneous velocity field. Similar structures were found earlier in turbulent pipe flow by Kim and Adrian (1999), who showed that the pre-multiplied energy spectra had two peaks occurring at $3 \delta$ and $14 \delta-20 \delta$, and that were called large-scale motions and very-large-scale motions (VLSMs) respectively. It has been observed in particle image velocimetry (PIV) experiments (Tomkins and Adrian 2003; Ganapathisubramani et al. 2003; Hambleton et al. 2006; Tang et al. 2012) that these highly elongated regions often exceed the streamwise extent of the PIV measurement region. With an attempt to capture the full extent of these regions, Hutchins and Marusic (2007a) deployed a spanwise rake of 10 hot-wire sensors and used Taylor's hypothesis (frozen convection) to reconstruct the instantaneous velocity field in a much larger streamwise domain, from which they discovered very long low- and high-speed regions that could occasionally exceed $15 \delta$ in length and named them as "superstructures". It has been shown that the VLSMs or superstructures make significant contributions to the turbulent kinetic energy (TKE) and the Reynolds shear stress (Liu et al. 2001; Ganapathisubramani et al. 2003; Guala et al. 2006; Hutchins and Marusic 2007a) and become more and more dominant in the turbulent structural landscape with increasing Reynolds number (Hutchins and Marusic 2007a,b). Additionally, the VLSMs have been found to strongly influence the near-wall cycle through superposition (Abe et al. 2004; Toh and Itano 2005; Hutchins and Marusic 2007a) and amplitude modulation of the underlying small-scale motions (Hutchins and Marusic 2007b; Marusic and Hutchins 2008; Mathis et al. 2009; Chung and McKeon 2010; Guala et al. 2011; Ganapathisubramani et al. 2012). Furthermore, it has been demonstrated that the large-scale streak-like features are associated with large-scale counter-rotating roll modes of similar length (Del Álamo et al. 2006; Marusic and Hutchins 2008; Hutchins et al. 2012).

Although knowledge on the features of VLSMs has recently expanded significantly, their origins remain an open question. For low Reynolds number flows, there are studies (Guala et al. 2006; Adrian 2007; Dennis and Nickels 2011; Lee and Sung 2011) that support the hypothesis that the bottom hairpin packets align coherently to form VLSMs, as suggested by Kim and Adrian (1999). At high Reynolds numbers, VLSMs tend to be mainly driven by "top-down" mechanisms (Hunt and Morrison 2000; Hunt and Carlotti 2001; Högström et al. 2002; Morrison 2007). Large-scale streak-like structures have also been found in the logarithmic layer of turbulent flows over fully rough surfaces, both in numerical studies and observational experiments (Coceal et al. 2007; Inagaki and Kanda 2010; Lu and Porté-Agel 2010; Mejia-Alvarez et al. 2014). In theory, the near-wall cycle is absent or strongly perturbed 
here due to the roughness elements. Hence these studies imply that the large-scale structures in the logarithmic and outer regions are not dependent on the small-scale near-wall structures.

Due to the advantages of having fully resolved temporal and spatial information and avoiding any modelling assumptions such as Taylor's hypothesis, direct numerical simulation (DNS) has been widely adopted to investigate various turbulent flows. Recently, DNS has also been increasingly used to explore VLSMs, and for such purpose the computational domain must be large enough to capture the large-scale structures properly. Priymak and Miyazaki (1994) performed DNS of pipe flow with the streamwise domain length $L_{x}=50 R$, where $R$ is the pipe radius, and found that the most energetic motion has a wavelength around $25 R$. However, the simulated pipe flow was not fully turbulent because the Reynolds number $\left(R e_{\tau}=150\right)$ was low. Recently, DNS of turbulent pipe flow at a relatively high Reynolds number with $L_{x}=30 R$ was achieved by Wu et al. (2012), and VLSMs of turbulent pipe and boundary-layer flows were compared by Lee and Sung (2013) through DNS. To investigate VLSMs in channel flow, Del Álamo et al. (2004) performed DNS at fully turbulent Reynolds numbers using numerical boxes with $L_{x}$ up to $8 \pi h$, where $h$ is the channel half-width. DNS of a turbulent boundary layer was recently performed by Lee and Sung (2011) to study the spatially coherent structures associated with VLSMs by using a computational box with $L_{x} \geq 50 \delta$. However, fully resolved DNS is computationally expensive (with cost proportional to $\operatorname{Re}_{\tau}{ }^{3} L_{x}$ ), and compared to other approaches its use in the study of VLSMs is limited to low Reynolds numbers. For reference, the most ambitious DNS of a channel flow to date is at $R e_{\tau}=2003$ and $L_{x}=8 \pi h$ (Hoyas and Jiménez 2006). DNS of VLSMs at higher $R e_{\tau}$ and larger $L_{x}$ is not yet possible.

Most laboratory studies of turbulent boundary layers have been conducted at low to moderate Reynolds numbers, $R e_{\tau} \sim \mathcal{O}\left(10^{3}-10^{4}\right)$; in contrast, atmospheric boundary-layer (ABL) flows are invariably at high Reynolds numbers, $R e_{\tau} \sim \mathcal{O}\left(10^{6}\right)$. Among the various types of $\mathrm{ABL}$ turbulence, the neutrally stable $\mathrm{ABL}$ is precisely connected to the laboratory flat-plate boundary layer according to the Reynolds number similarity. In both of them, thermal effects are absent and turbulence is shear-driven with an approximate logarithmic velocity profile near the surface. Unlike the low Reynolds number boundary layer in which the turbulence is dominated by the near-wall cycle, the inner and outer scales in the ABL are well separated with the logarithmic region occupying almost the entire layer and its associated structures dominating the turbulence. In the last few decades, there are numerous experimental and numerical studies on the large-scale streaks in the neutral ABL (Wilczak and Tillman 1980; Moeng and Sullivan 1994; Boppe and Neu 1995; Khanna and Brasseur 1998; Carlotti 2002; Young et al. 2002; Drobinski et al. 2004; Foster et al. 2006; Dubos et al. 2008; Newsom et al. 2008; Horiguchi et al. 2010; Lu and Porté-Agel 2010). Recently, experimental field studies on the VLSMs in the ABL have emerged. Using measurements made at the Surface-Layer Turbulence and Environmental Science Test facility in the salt flats of Utah under near-neutral conditions, Guala et al. (2011) investigated interactions between VLSMs and the near-wall turbulent structures, and Hutchins et al. (2012) examined the presence of VLSMs in the $\mathrm{ABL}$, comparing the large-scale coherence in the $\mathrm{ABL}$ and in the laboratory-scale boundary layer. However, it is known that field measurements are more challenging than laboratorybased measurements, and can lack resolution and statistical convergence. Such challenges have implications on the robustness of the analysis, which may be further adversely affected by the use of Taylor's hypothesis. Dennis and Nickels (2008) pointed out that using Taylor's hypothesis can lead to incorrect interpretations for large-scale structures in a turbulent boundary layer. Using DNS data of a turbulent channel flow, Del Álamo and Jiménez (2009) examined the application of Taylor's hypothesis to experiments and found that this technique can lead to an artificial secondary peak in the energy spectrum. 
Large-eddy simulation (LES) has become an important tool for investigating turbulent transport phenomena in the ABL. This is largely due to the fact that LES is superior to the conventional Reynolds-averaged Navier-Stokes (RANS) approach in predicting unsteady turbulent behaviors and computationally more efficient than DNS. In LES, only large eddies contributing to the TKE production (i.e. larger than the filter size) are calculated and the net effects of small eddies (i.e. smaller than the filter size) are parametrized using suitable subgrid-scale (SGS) schemes. Therefore practical grid sizes much larger than those required to resolve all of the fluid motions can be used and the computational cost is dramatically reduced compared to DNS. This makes LES ideally suitable for the study of VLSMs, though at the cost of introducing SGS and wall-modelling errors. Nevertheless, a recent numerical simulation of long channel flow (Chung and McKeon 2010) has shown that a controlled application of LES equipped with a suitable SGS model is able to capture the essential features of these large-scale structures. LES has already been used to study the neutral ABL, e.g., Andren (1994), Moeng and Sullivan (1994), Ayotte et al. (1996), Lin et al. (1996), Albertson and Parlange (1999), Porté-Agel et al. (2000), Ding et al. (2001), Bou-Zeid et al. (2004), Bou-Zeid et al. (2005), Stoll and Porté-Agel (2006), Foster et al. (2006), Dubos et al. (2008), Finnigan et al. (2009), Lu and Porté-Agel (2010). Most of these studies focus on time-averaged and horizontally-averaged vertical profiles of relevant physical quantities, and hence use rather short streamwise domain sizes $\left(L_{x} \leq 2 \pi L_{z}\right)$. To our knowledge, LES has not yet been used to investigate the VLSMs in the neutral ABL.

The purpose of the present study is to use LES with a large computational domain to properly assess features of VLSMs in the neutral ABL and compare the simulation results with recent experimental findings. Details of the simulations are given in the next section. Results are presented and discussed in Sect. 3, with conclusions in Sect. 4.

\section{Simulation Details}

We use the WIRE-LES model, which has been developed from the original version described in Albertson and Parlange (1999) and Porté-Agel et al. (2000). The code solves the threedimensional filtered Navier-Stokes equations written in rotational form

$$
\begin{aligned}
\frac{\partial \widetilde{u}_{i}}{\partial x_{i}} & =0 \\
\frac{\partial \widetilde{u}_{i}}{\partial t}+\widetilde{u}_{j}\left(\frac{\partial \widetilde{u}_{i}}{\partial x_{j}}-\frac{\partial \widetilde{u}_{j}}{\partial x_{i}}\right) & =-\frac{\partial \widetilde{p}^{*}}{\partial x_{i}}-\frac{\partial \tau_{i j}}{\partial x_{j}}+F_{i},
\end{aligned}
$$

where $t$ denotes time, $\widetilde{u}_{i}$ is the $i$ th component of the instantaneous resolved velocity, $x_{i}$ is the $i$ th component of the position vector, $\widetilde{p}^{*}=\widetilde{p} / \rho+\frac{1}{2} \widetilde{u}_{i} \widetilde{u}_{i}$ is the modified pressure divided by density $\rho$ and including the resolved kinetic energy, $\tau_{i j}$ is the SGS stress tensor, and $F_{i}$ is the component of a body force. The viscous term is neglected in Eq. 2 because the Reynolds number of the ABL flow is high and the dissipation is dominated by the SGS term. Since the focus here is on the neutral ABL, no additional terms are used to account for buoyancy effects. The flow is driven by a constant pressure gradient $F_{i}$ in the streamwise direction (the Coriolis force has not been considered).

The quality of the SGS model used to represent the unresolved motions is critical for accurate calculations, especially for our purpose here to capture the dynamics of the large-scale structures. We use the well-tested Lagrangian-averaged scale-dependent (LASD) dynamic model (Stoll and Porté-Agel 2006), which belongs to the family of the Smagorinsky models. 
The dynamic procedure eliminates both the need of tuning the Smagorinsky model coefficient and the questionable assumption that a single value of the Smagorinsky coefficient is valid for any simulation. Additionally, the LASD model does not rely on the scale-invariance assumption of the standard dynamic Smagorinsky model (i.e. the Smagorinsky model coefficient is the same at different scales). Instead, it uses a second test-filtering operation to determine how the coefficient changes across scales, thus providing more accurate estimation of the coefficient at the grid scale. Compared to the traditional Smagorinsky model and the standard dynamic model, the scale-dependent dynamic model yields improved dissipation characteristics, velocity spectra, and mean velocity profiles in simulations of the neutral ABL (Porté-Agel et al. 2000). Moreover, the Lagrangian time-averaging approach (Meneveau et al. 1996) is adopted in the LASD model to reduce noise and maintain numerical stability for applications in complex geometry flows with statistical inhomogeneity. The LASD model has been shown to be particularly well suited to capturing unresolved small-scale turbulence in complex environments in which SGS turbulence deviates from the classical assumptions of isotropic, inertial-range behaviour (Bou-Zeid et al. 2005; Stoll and Porté-Agel 2006, 2008).

Periodic boundary conditions are applied for both streamwise and spanwise directions. The upper boundary is specified as a frictionless rigid lid, i.e. a stress-free condition. The lower boundary condition is formulated using Monin-Obukhov similarity theory, in which the instantaneous wall-shear stress is related to the velocity at the first grid point above the boundary through the logarithmic law with a specified aerodynamic roughness length $z_{0}$.

Strictly speaking, the stress-free condition at the top is only valid for either a free boundary layer or a neutral ABL under the influence of the Coriolis force. Nevertheless, in LES of the neutral ABL without Coriolis effects, it is common practice to use the stress-free condition as well at the upper boundary of the simulation domain (Shaw and Schumann 1992; Albertson and Parlange 1999; Porté-Agel et al. 2000; Bou-Zeid et al. 2005; Finnigan et al. 2009; Lu and Porté-Agel 2010). Physically, it can be interpreted as a strong capping inversion, which prevents further deepening of the boundary layer. Moreover, due to the periodic condition applied at the lateral boundaries, no boundary-layer growth is allowed. Hence, the height of the simulation domain essentially defines the boundary-layer depth $\delta$. Even though the stress-free condition cannot represent the entrainment of free atmosphere momentum into the boundary layer, the effect on the turbulence statistics is mainly restricted to the region near the upper boundary.

The computational domain is defined by the three scales $L_{x}, L_{y}$, and $L_{z}$ along the streamwise, spanwise, and vertical (wall-normal) directions and discretized using $N_{x}, N_{y}$ and $N_{z}$ nodes, respectively. In the streamwise and spanwise directions all derivatives are computed by the pseudospectral method, while in the vertical direction derivatives are computed with second-order centered finite differences. All non-linear terms are de-aliased in Fourier space by the $3 / 2$ rule; the second-order Adams-Bashforth scheme is used for time marching. The incompressibility of the fluid expressed by Eq. 1 is satisfied by the projection method, in which the derived Poisson equation is solved for the pressure field, which is then used to correct the velocity field to become divergence free. The test filters used in the dynamic procedure are two-dimensional low-pass cut-off filters that take advantage of the spectral nature of the code in the horizontal directions. Previous studies (Porté-Agel et al. 2000; Porté-Agel 2004; Basu and Porté-Agel 2006; Stoll and Porté-Agel 2006, 2008; Kleissl et al. 2006; Kumar et al. 2006; Anderson et al. 2012; Abkar and Porté-Agel 2012; Cancelli et al. 2014) demonstrate that the LES model reproduces well-known features of the quasi-steady boundary layer under various stability conditions and also the entire 24-h diurnal evolution of the ABL. 
To capture VLSMs properly, we use a large computational domain with $L_{z}=1000 \mathrm{~m}$, $L_{y}=4 \pi L_{z}$, and $L_{x}=32 \pi L_{z}$. The simulations are carried out using two grid resolutions with $N_{x} \times N_{y} \times N_{z}=1024 \times 128 \times 64$ and $2048 \times 256 \times 128$ respectively, with a uniform grid $\left(\Delta_{x}=\Delta_{y}=2 \pi \Delta_{z}\right)$ throughout the simulation domain. The imposed pressure gradient $F_{x}$ is specified as $U_{\tau}^{2} / L_{z}$, in which the friction velocity $U_{\tau}$ is set to $0.45 \mathrm{~m} \mathrm{~s}^{-1}$. The roughness length $z_{0}$ is taken to be $0.1 \mathrm{~m}$, and the timestep is set to $0.2 \mathrm{~s}$ in order to meet the Courant-Friedrichs-Lewy stability condition and the viscous stability. A warm-up simulation is firstly made for a long enough time to guarantee that quasi-steady conditions are reached (i.e. the profile of the total stress normalized by $U_{\tau}^{2}$ adjusts to a straight line reaching 1 at the surface and the mean resolved kinetic energy becomes stable). Statistical calculations are then performed over $2 \times 10^{6}$ timesteps to ensure statistical convergence. The main features of VLSMs obtained with the coarse and fine resolutions are qualitatively the same. Therefore, only the results obtained with the fine resolution are shown herein, and quantitative differences between the results obtained with the two resolutions are commented upon when necessary.

\section{Results}

\subsection{Instantaneous Fields}

Figure 1 shows the fluctuations of the streamwise velocity component normalized by the friction velocity in the horizontal plane at $z / \delta=1 / 8$. The flow is from left to right. The filled 2 -D contour plot of the instantaneous fluctuations at a given time $t_{s}$ after reaching the quasisteady state is presented in Fig. 1a, which shows typical features in the logarithmic region of a turbulent boundary layer. Several very long low-speed streaks with negative fluctuations (marked by dark grey contours) meandering in the downstream direction can be seen clearly. Each of these low-speed streaks is flanked on either side by similarly elongated high-speed streaks with positive fluctuations (marked by light grey contours). The majority of these streaks has length more than $5 \delta$ in the streamwise direction. These patterns are similar to the VLSMs observed in both experimental and DNS studies of turbulent boundary layers (Hutchins and Marusic 2007a; Lee and Sung 2011), as well as field measurements of ABL flows (Hutchins et al. 2012).

To highlight the characteristics of very long structures, we applied a two-dimensional low-pass filter to the instantaneous velocity field. Based on the spanwise correlation of the streamwise velocity component shown in Fig. 9, a filter size of $0.4 \delta$ was chosen, which is close to the spanwise correlation length at $z / \delta=1 / 8$. So, velocity fluctuations at scales smaller than the characteristic spanwise width of the elongated structures are removed by the filter. Figure $1 \mathrm{~b}-\mathrm{d}$ shows the fluctuations of the filtered streamwise velocity component at three different time intervals in the same horizontal plane as in Fig. 1a. The time interval here is normalized by the characteristic time $T=L_{z} / U_{\tau}$, which can be regarded as the large-eddy turnover time; note that Fig. $1 \mathrm{a}$ and $\mathrm{b}$ are for the same time. Positive and negative fluctuations are represented by grey and white colours, respectively. The low-speed regions are elongated in the streamwise direction and can reach $20 \delta$ in length, while their characteristic width is around $0.5 \delta$ in the spanwise direction. The time evolution of the filtered fields shows that, during a time period of $0.18 T$, the VLSMs travel downstream with very little change in their forms (i.e., the dispersion is small). Note that a long lifespan comparable to the large-eddy turnover time is a major prerequisite for a coherent structure, and the dispersion must be small in order for the coherent structure to be long-lived (Adrian 2007). 


\begin{tabular}{lllllll|}
-6 & -4 & -2 & 0 & 2 & 4 & 6 \\
\hline & 1 & 1 & 1 & 1 & \\
\hline
\end{tabular}

(a) $t_{s}$

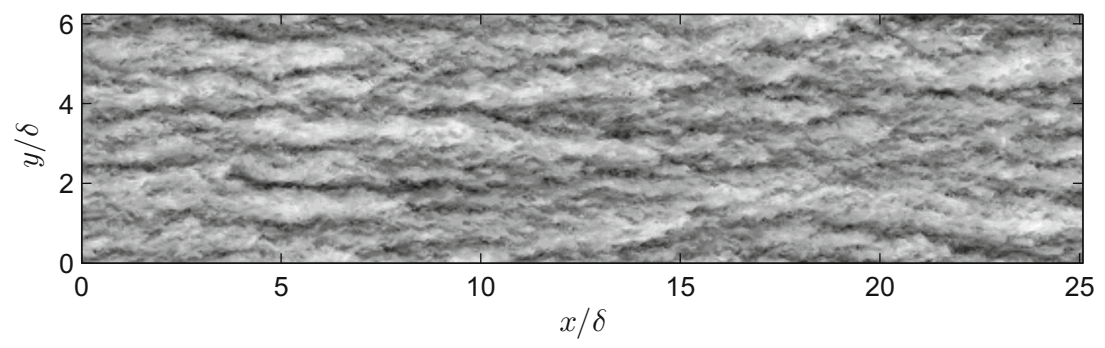

(b) $\left(t-t_{s}\right) / T=0$

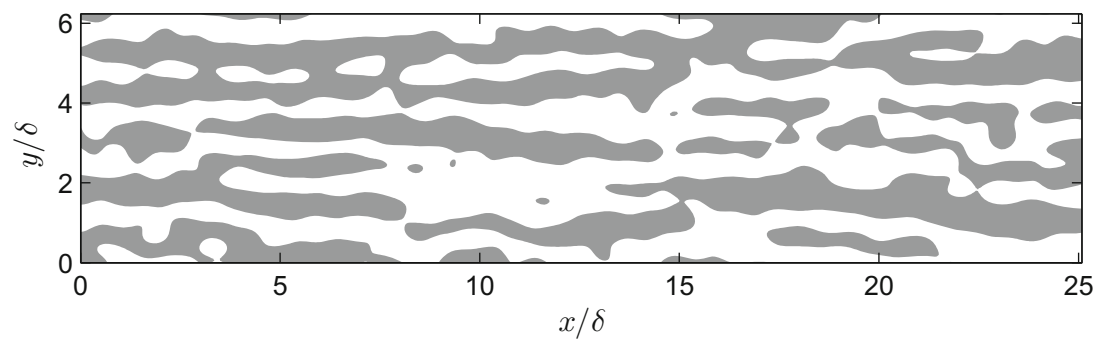

(c) $\left(t-t_{s}\right) / T=0.09$

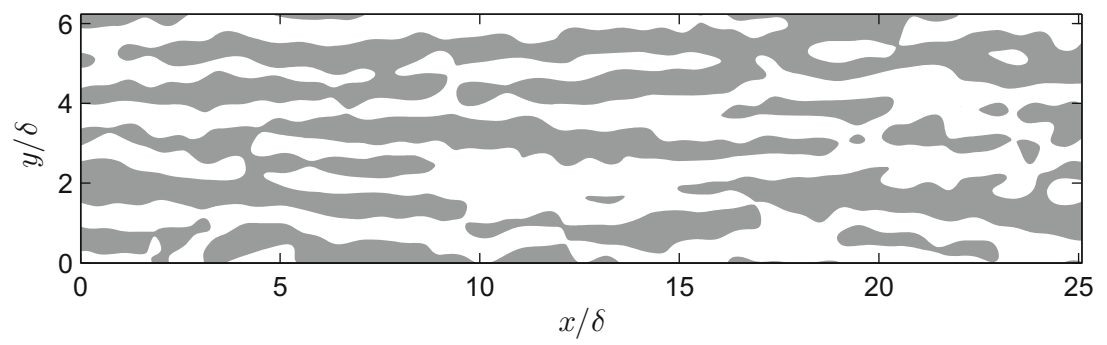

(d) $\left(t-t_{s}\right) / T=0.18$

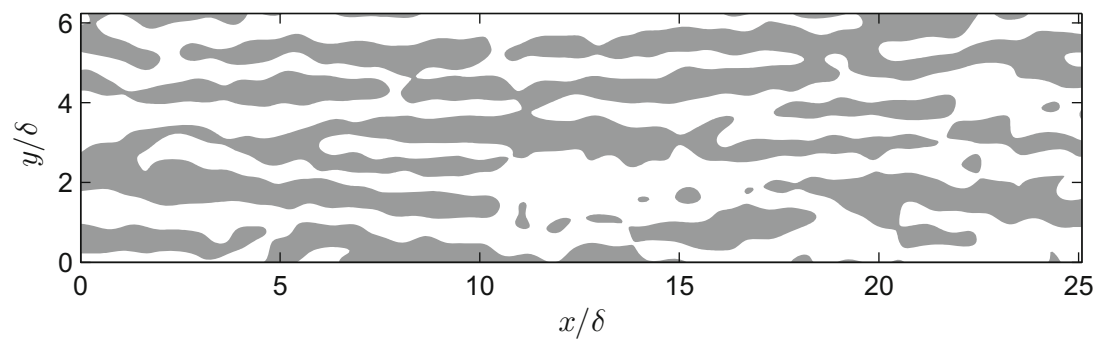

Fig. 1 Fluctuations of the streamwise velocity component normalized by the friction velocity in the horizontal plane at $z / \delta=1 / 8$. a The instantaneous field at a given time $t_{s} ; \mathbf{b}-\mathbf{d}$ The spatially-filtered fields at $\left(t-t_{S}\right) / T=$ $0,0.09,0.18$, respectively 
(a) $z / \delta=1 / 32$

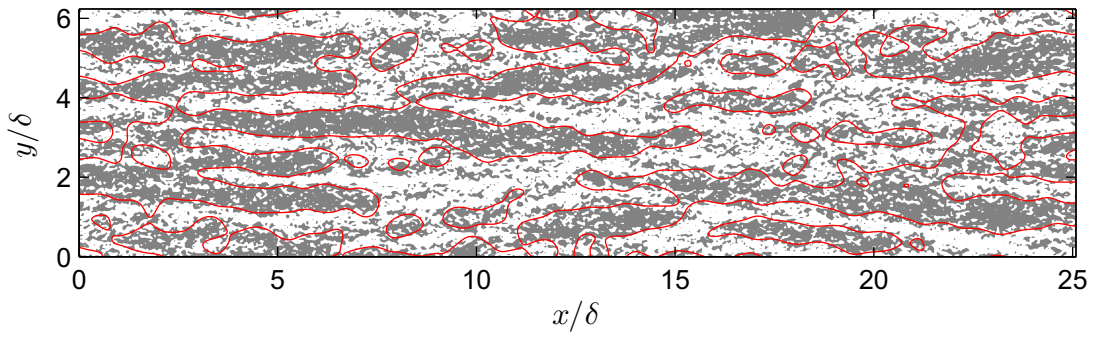

(b) $z / \delta=1 / 16$

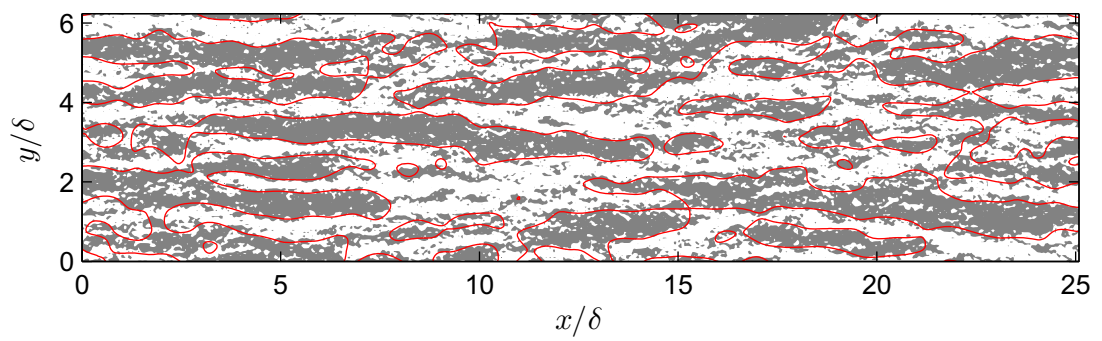

(c) $z / \delta=3 / 16$

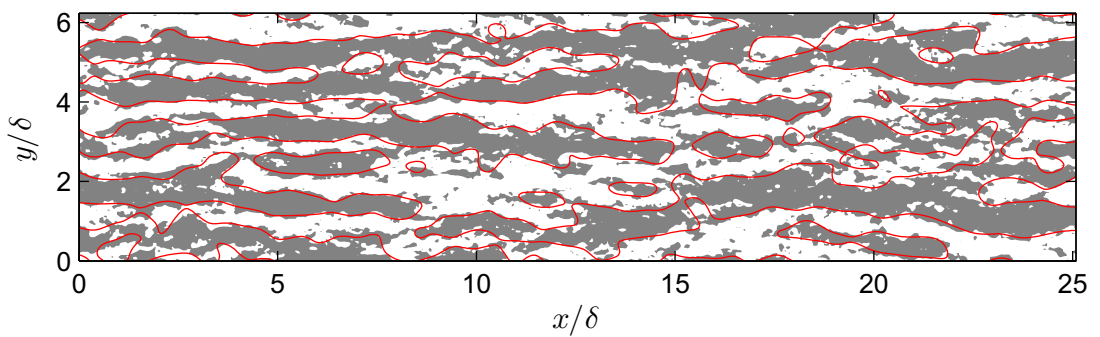

Fig. 2 Instantaneous fluctuations of the streamwise velocity component normalized by the friction velocity in the horizontal planes at three different heights: $\mathbf{a} z / \delta=1 / 32 ; \mathbf{b} z / \delta=1 / 16 ; \mathbf{c} z / \delta=3 / 16$. Positive and negative fluctuations are represented by dark and white colours, respectively. In each sub-figure, the red contour line is for the spatially-filtered field and corresponds to the value of zero

The contour plots of the fluctuations of the streamwise velocity component in the horizontal plane at different distances from the wall are shown in Fig. 2. It can be observed that the continuation of the near-wall structures shown in Fig. 2a is less than that of the outer ones shown in Fig. $2 b$ and c. In addition, the large-scale features of Fig. $2 b$ and $c$ have a superimposed footprint in the small-scale structures of Fig. 2a, which implies that smallscale motions near the wall are strongly influenced by large-scale motions from above. This characteristic is highlighted by applying the same spatial filter as before. It can be seen that the red contour lines for the filtered fluctuation fields in Fig. $2 \mathrm{a}-\mathrm{c}$ are quite similar.

In Fig. 3, the upper sub-figure shows the instantaneous fluctuations of the spatially-filtered spanwise velocity component normalized by the friction velocity in the $x-z$ planes at two spanwise locations of $y=9.95 \delta$ and $y=10.45 \delta$. The lower sub-figure shows the instantaneous fluctuations of the spatially-filtered streamwise velocity component normalized by the friction velocity in the horizontal plane at $z=0.125 \delta$. The two dashed lines show the loca- 


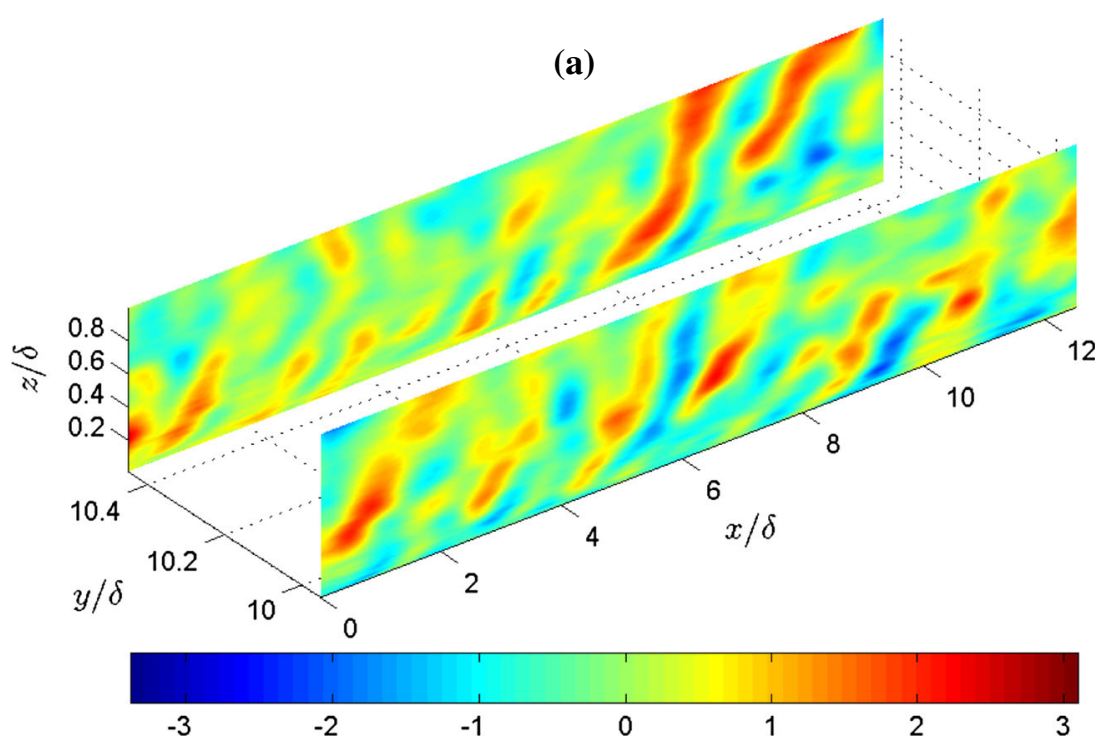

(b)

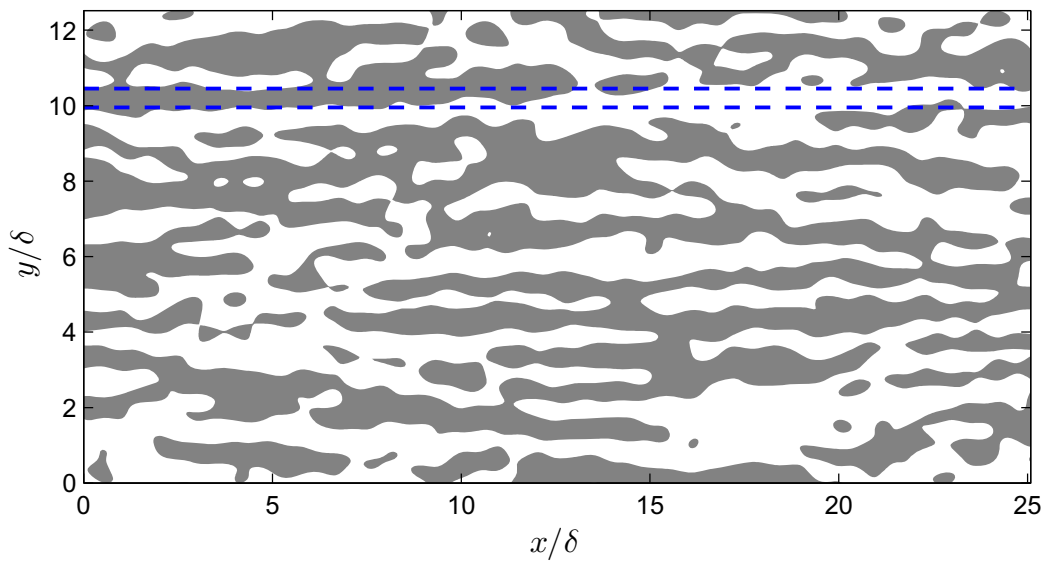

Fig. 3 a Instantaneous fluctuations of the spatially-filtered spanwise velocity component normalized by the friction velocity in the $x-z$ planes at two locations of $y=9.95 \delta$ and $y=10.45 \delta$; $\mathbf{b}$ instantaneous fluctuations of the spatially-filtered streamwise velocity component normalized by the friction velocity in the horizontal plane at $z=0.125 \delta$ with positive and negative fluctuations represented by dark and white colours, respectively. The two dashed lines in plot (b) show the locations of the two $x-z$ planes in plot (a)

tions of the two $x-z$ planes (shown in Fig. 3a) inside the horizontal plane. For the spanwise velocity component, there are distinctive inclined regions of positive and negative fluctuations in the $x-z$ planes, which stretch all the way from the wall to the top of the boundary layer. An inclined region of positive fluctuations is often followed by an inclined region of negative fluctuations, and vice versa. A superstructure event in the streamwise velocity component is accompanied by the inclined structures in the spanwise velocity component on the two sides. This pattern can be clearly observed by pairing the two sub-figures of Fig. 3 in the range of $x$ from 0 to $10 \delta$; we also note that a pair of inclined structures flanking a superstructure 
event (here a high-speed one) have the opposite fluctuation to the spanwise velocity component. This observation suggests that a VLSM is associated with counter-rotating rolls and/or inclined vortices. It is worth mentioning that the results in Fig. 3 are very similar to the results for the spanwise velocity component presented in Fig. 12 of Hutchins et al. (2012).

\subsection{Statistical Analysis}

In the previous sub-section, the plots of the instantaneous velocity fields show that VLSMs exist in the logarithmic and outer regions of the neutral ABL in the form of alternating lowand high-speed streaks. Those results reveal also some qualitative features of the VLSMs. In this sub-section, characteristics of the VLSMs are further elucidated from a statistical point of view.

First, the pre-multiplied power spectrum is used to obtain the characteristic sizes of large and very large structures. Here, $E_{u u}$, the power spectrum of a velocity component $u$, is multiplied by the wavenumber $k$ to yield $k E_{u u}$. In the semi-logarithmic plot of $k E_{u u}$ (with a linear scale) versus $k$ (with a logarithmic scale), the area under the curve corresponds to the kinetic energy of the velocity component $u$. Hence such a plot illustrates clearly the contributions of different wavelengths to the kinetic energy and helps to find the dominant wavelengths by locating the peaks.

Figure 4 presents the pre-multiplied power spectra as functions of the streamwise wavelength $\lambda_{x}$ for the streamwise velocity component at various heights. Each pre-multiplied power spectrum shows a bimodal or plateau distribution with the short wavelength peak corresponding to large scale motions and the long wavelength peak corresponding to VLSMs. For $z \leq 0.125 \delta$, with increasing distance from the wall, the wavelength at which the plateau starts increases considerably from $0.5 \delta$ to $3 \delta$, while the wavelength at which the plateau ends increases slightly. This is consistent with the instantaneous flow patterns seen in Fig. 2. This implies that more of the TKE is carried by the VLSMs in comparison with the largescale motions and the smaller ones. For $z>0.125 \delta$, the wavelengths of the two peaks and the ratio of the long wavelength peak to the short wavelength peak remain nearly constant as $z$ increases. The wavelength of the second peak, which represents the streamwise size of VLSMs, is between $10 \delta$ and $30 \delta$. This is consistent with the experimental finding of Hutchins and Marusic (2007a), who showed that very long meandering structures are present in the logarithmic region of the neutrally stable ABL and have a streamwise length of $O(20 \delta)$. It is worth mentioning that Fig. 4 shows a spectral gap between large-scale motions and VLSMs for $z>0.125 \delta$. This spectral gap separates two types of turbulent motions, and, therefore, it is different from the traditional one, which separates the energy contribution of turbulent motions from that of mesoscale motions (Van der Hoven 1957).

For completeness, the pre-multiplied power spectra for the spanwise and vertical velocity components are shown in Figs. 5 and 6 respectively. For the spanwise velocity component, the pre-multiplied power spectrum at each height has only one peak, with the corresponding wavelength increasing with increasing height and reaching a maximum around $\delta$. The premultiplied power spectra for the vertical velocity component are qualitatively similar to those for the spanwise velocity component. In the coarse resolution simulation, it was observed that, for each velocity component, the pre-multiplied power spectra present more pronounced short wavelength peaks for $z \leq 0.125 \delta$.

The pre-multiplied cospectra of $u$ and $w$ as functions of the streamwise wavelength $\lambda_{x}$ at different heights are calculated and presented in Fig. 7. Each pre-multiplied cospectrum has a distribution similar to the pre-multiplied power spectrum for the streamwise velocity component, but with a less pronounced second peak. With increasing distance from the wall, 


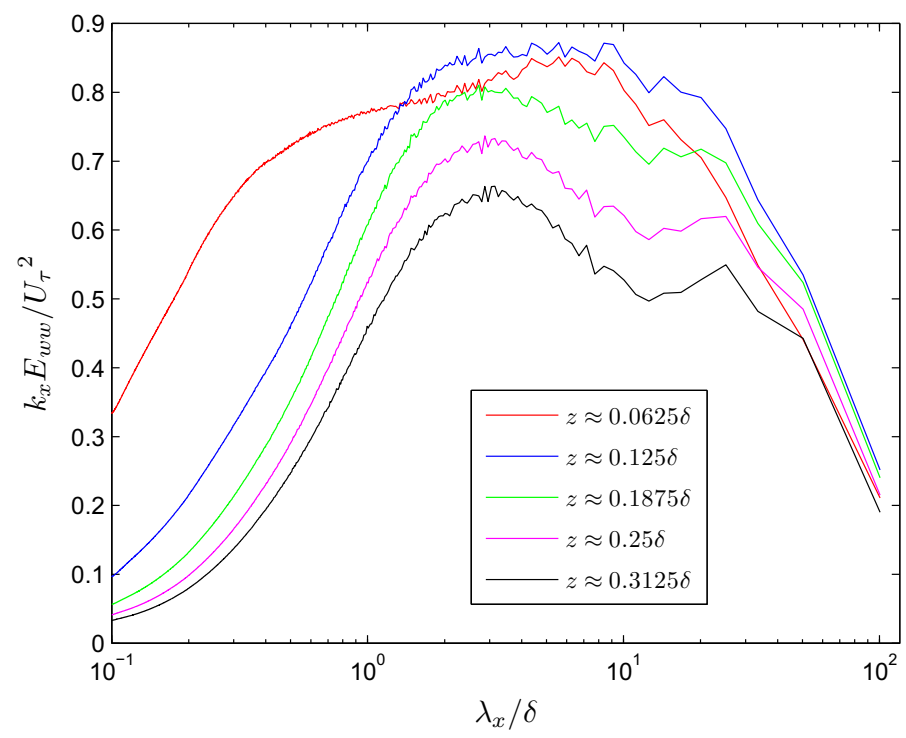

Fig. 4 Pre-multiplied power spectra as functions of the streamwise wavelength for the streamwise velocity component at various heights

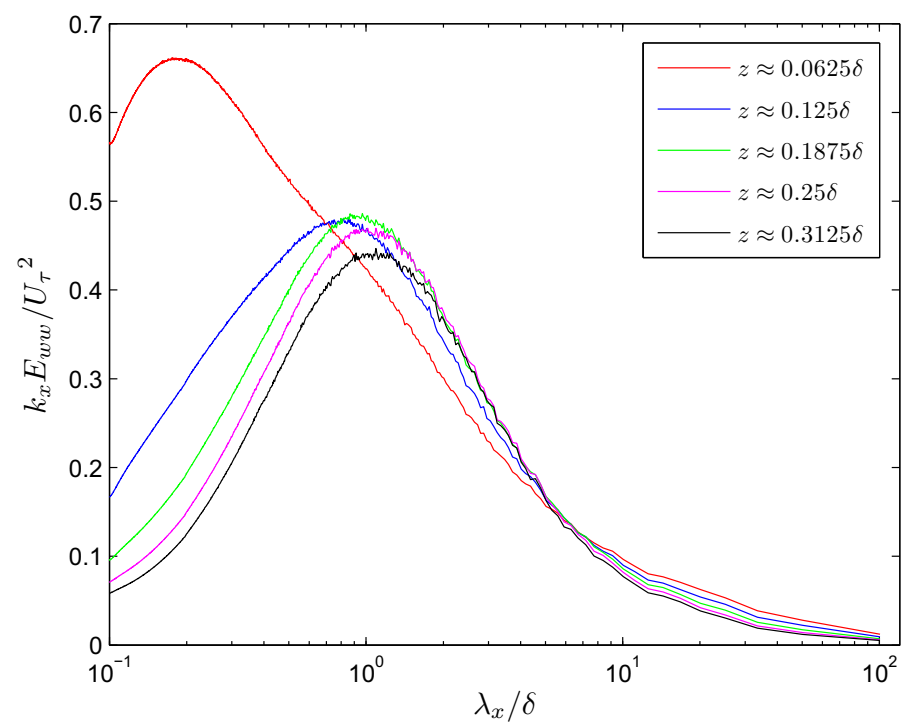

Fig. 5 Pre-multiplied power spectra as functions of the streamwise wavelength for the spanwise velocity component at various heights

the changing trend of the pre-multiplied cospectrum is similar to that of the pre-multiplied power spectrum for the streamwise velocity component, which indicates that the contributions to the vertical momentum flux from VLSMs become increasingly important.

By integrating the power spectra of the three velocity components and the cospectra of $u$ and $w$ from a short wavelength to a long wavelength, the contributions to the resolved TKE and 


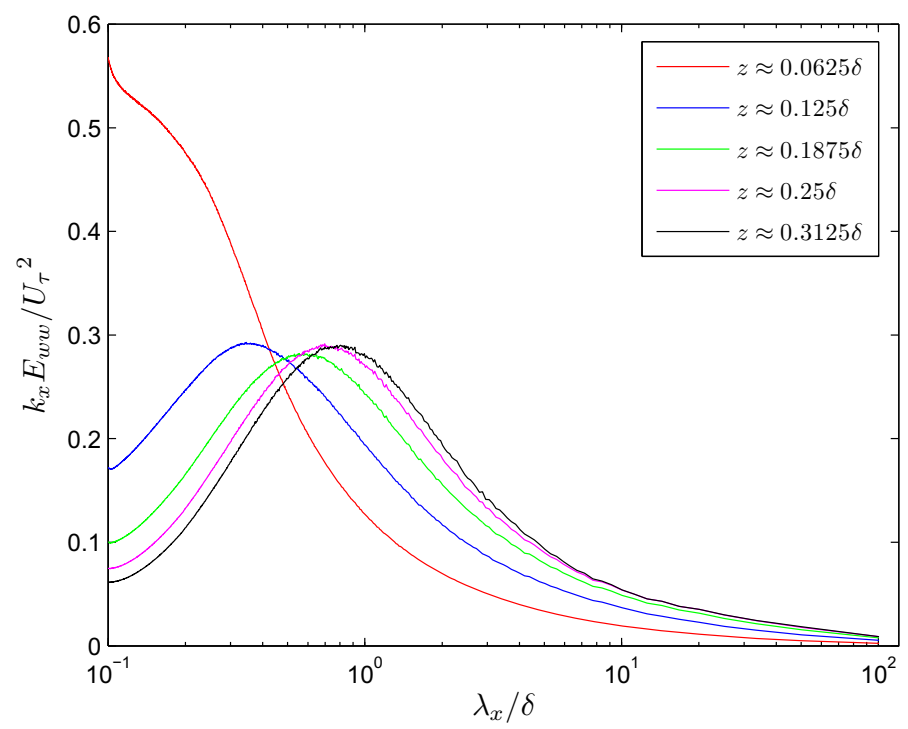

Fig. 6 Pre-multiplied power spectra as functions of the streamwise wavelength for the vertical velocity component at various heights

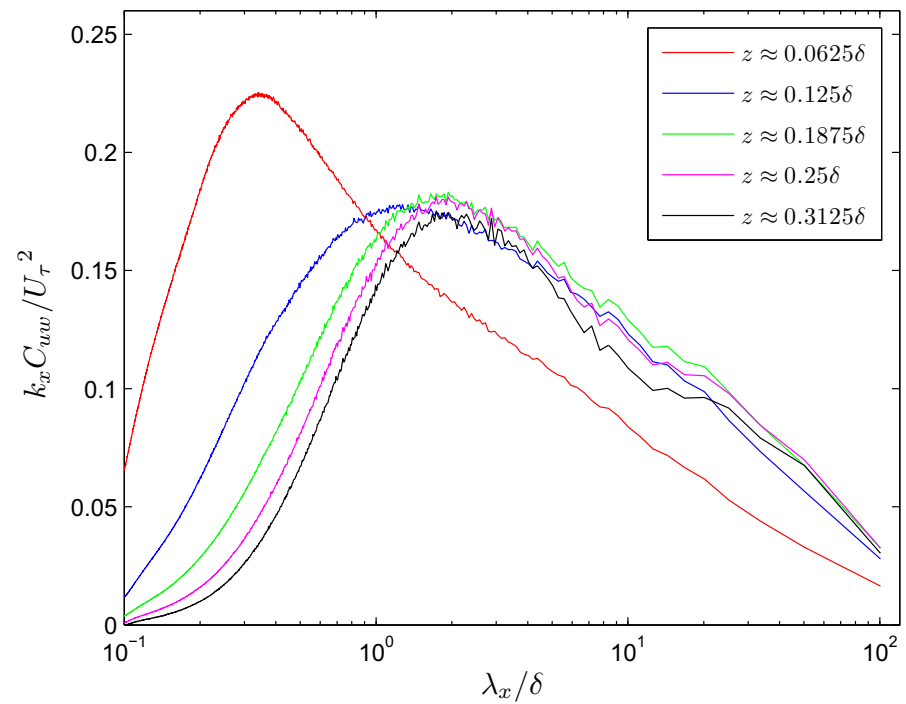

Fig. 7 Pre-multiplied cospectra of the streamwise and vertical velocity components as functions of the streamwise wavelength at various heights

shear stress from that range of wavelengths can be obtained. It is found that the contributions to the resolved TKE and shear stress from streamwise wavelengths larger than $10 \delta$ increase with height and reach a maximum of 27 and $31 \%$ respectively. This finding confirms that the large computational domain adopted here is essential for the purpose of investigating VLSMs. On the other hand, it suggests that the standard domain size ( $L_{x}=L_{y}=2 \pi L_{z}$ or even smaller), used in previous LES-based studies of ABL flows, could lead to unphysical 


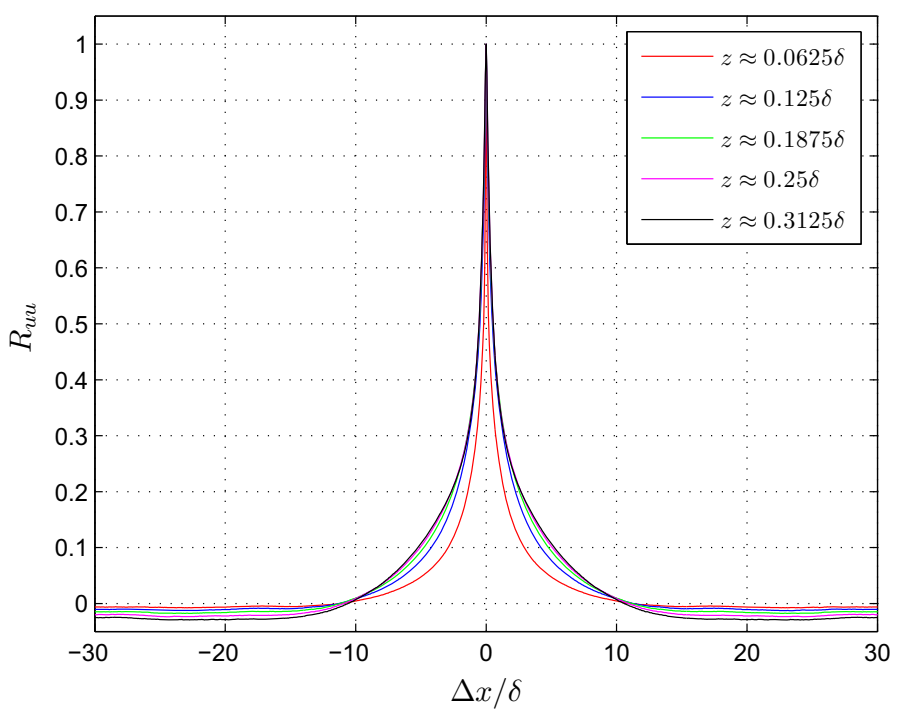

Fig. 8 Streamwise two-point correlation coefficients of the streamwise velocity component $R_{u u}(\Delta x, 0, z)$ calculated at different distances from the wall

results due to the truncation of the important VLSMs. This could explain, for example, the presence of streak structures occupying the entire streamwise domain and being locked-in in the spanwise position and in time (Lu and Porté-Agel 2010), which leads to large spanwise variations of the mean velocity (Finnigan et al. 2009). This unphysical effect has also been found in this study when using the standard domain size (results not shown here). It is worth mentioning that some recent studies (Ludwig et al. 2009; Mirocha et al. 2010; Kirkil et al. 2012; Mirocha et al. 2013) have suggested that streak structures could be artificially elongated when using relatively coarse resolutions and/or low-accuracy numerical schemes and SGS models. This could further worsen the locking effect.

Next, the two-point correlation coefficient for the streamwise velocity component $\left(R_{\text {ии }}(\Delta x, \Delta y, z)\right)$ is employed to further characterize the coherent structures. Figure 8 shows $R_{u u}$ in the streamwise direction with $\Delta y=0$ and various values of $z$. The correlations decay slowly, e.g., the correlation is still around 0.1 at $\Delta x= \pm 5 \delta$ for $z=0.25 \delta$, and is a sign of the presence of very long streamwise-elongated structures. A visual comparison of the correlations shown in Fig. 8 reveals that, with increasing distance from the wall, the streamwise correlation length increases when $z \leq 0.25 \delta$ and remains nearly constant when $z>0.25 \delta$. It is also observed that the correlations are positive when $|\Delta x| \leq 10 \delta$ and become slightly negative afterwards. This indicates the existence of very long structures followed by similar ones but with opposite fluctuations, which is consistent with the instantaneous flow pattern seen in Fig. 1.

Figure 9 shows $R_{u u}$ in the spanwise direction with $\Delta x=0$ and various values of $z$. Here, the first interesting observation is that the correlations show a strong alternating positivenegative trend, which is indicative of alternating low- and high-speed streaks shown in Figs. 1 and 2. Secondly, it can be observed that the spanwise width of the correlation increases as the height increases. This indicates a spanwise growth of coherent structures along the wall-normal direction, which is in support of the hairpin-concatenation hypothesis of Kim and Adrian (1999). Lastly, approximately within the range of $-0.25 \delta \leq \Delta y \leq 0.25 \delta$, the streamwise velocity component remains positively correlated, from which the spanwise 


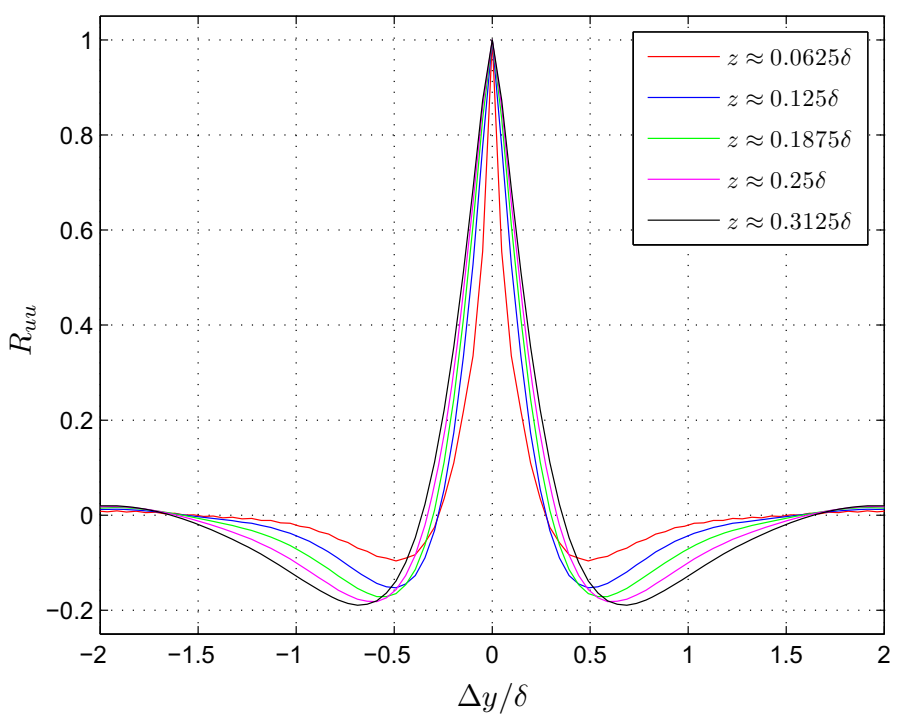

Fig. 9 Spanwise two-point correlation coefficients of the streamwise velocity component $R_{u u}(0, \Delta y, z)$ calculated at different distances from the wall

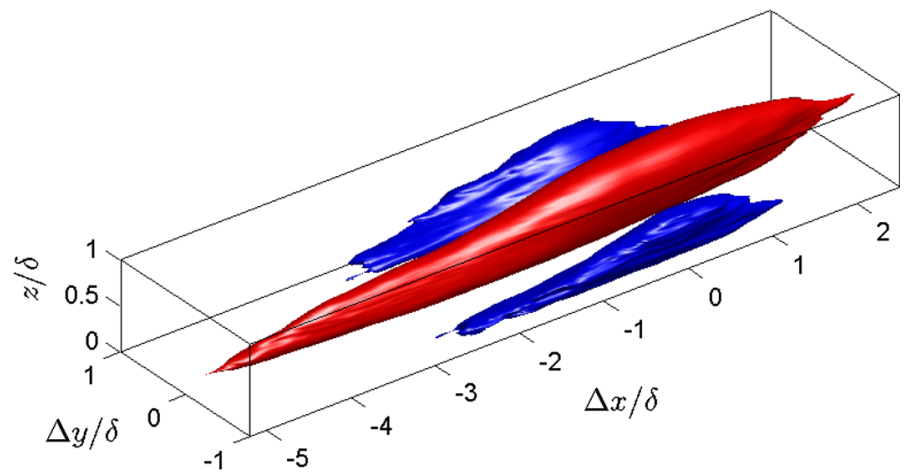

Fig. 10 Iso-surfaces of $R_{u u}^{3 \mathrm{~d}}$ with $z_{\text {ref }}=\delta / 2$. Red iso-surfaces show positive correlation $\left(R_{u u}^{3 \mathrm{~d}} \geq 0.25\right)$; blue iso-surfaces show negative correlation $\left(R_{u u}^{3 \mathrm{~d}} \leq-0.15\right)$

width of the streaks is estimated to be $0.5 \delta$. It is worth mentioning that the characteristic streamwise length and spanwise width of VLSMs obtained by the fine resolution simulation are consistent with those reported in previous studies of laboratory-scale boundary layers, while those obtained by the coarse resolution simulation are relatively larger. Preliminary results (not shown here) obtained with an even higher resolution have shown little differences with respect to the results presented here.

For a general analysis of VLSMs in three dimensions, we introduce the 3D spatial correlation function defined as

$$
R_{u u}^{3 \mathrm{~d}}\left(\Delta x, \Delta y, z, z_{\mathrm{ref}}\right)=\frac{\left\langle u^{\prime}\left(x, y, z_{\mathrm{ref}}\right) u^{\prime}(x+\Delta x, y+\Delta y, z)\right\rangle}{\left\langle u^{\prime 2}\left(x, y, z_{\mathrm{ref}}\right)\right\rangle}
$$

for the streamwise velocity component. Figure 10 shows the iso-surfaces of $R_{u u}^{3 \mathrm{~d}}$ with $z_{\text {ref }}=\delta / 2$. The region of positive correlation (marked by red) is highly elongated in the 


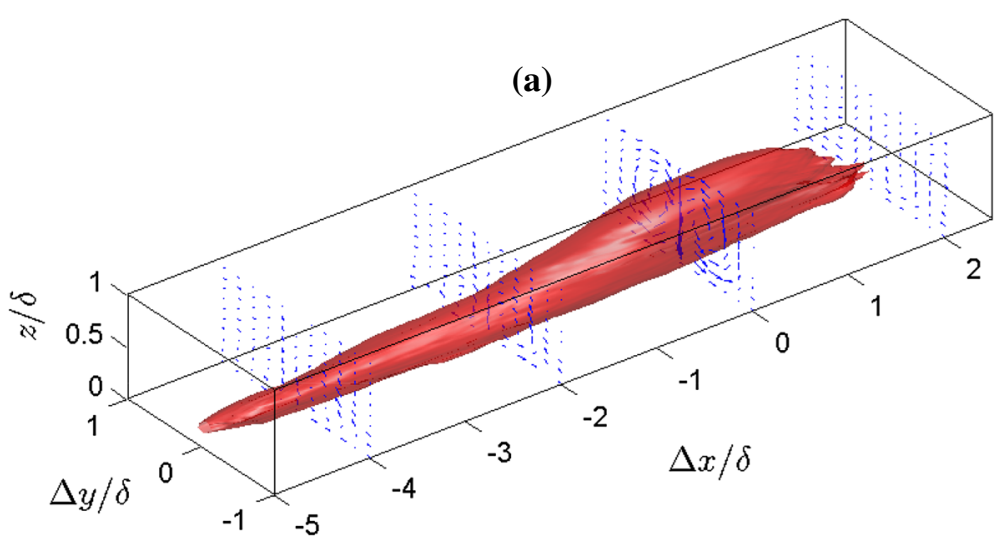

(b)

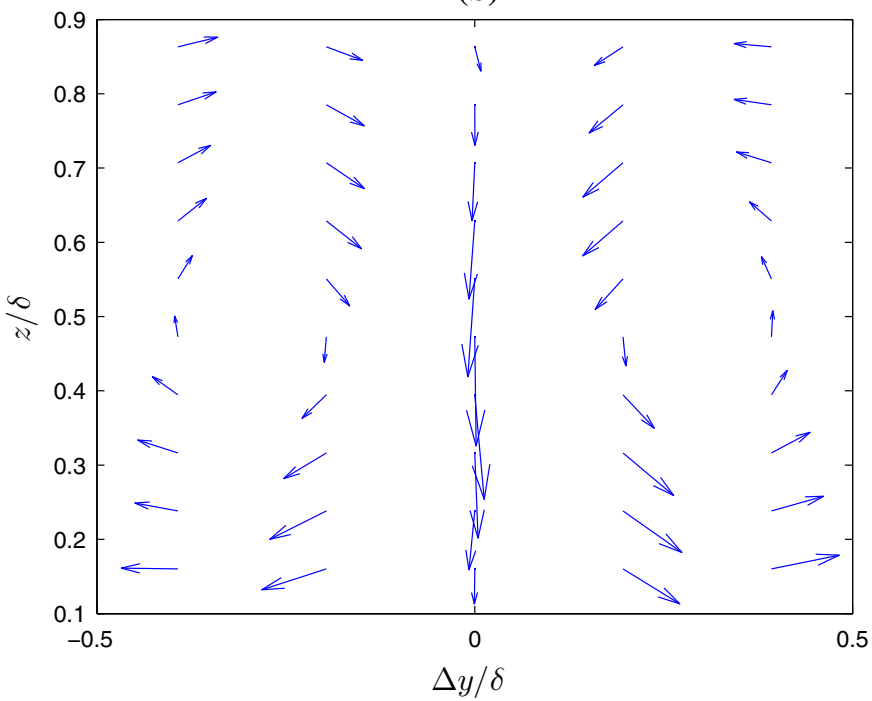

Fig. 11 Conditionally-averaged flow field. The conditional event is a high-speed event (of positive $u$ fluctuation) at $\Delta x=0, \Delta y=0, z=\delta / 2$. a Red iso-surfaces show positive $u$ fluctuation ( $\geq 0.3)$; vector fields for the velocity components $v$ and $w$ are shown on spanwise/wall-normal planes at $\Delta x / \delta=-4,-2,0$, and 2. b Enlarged view of the plot of the vector field on the spanwise/wall-normal plane at $\Delta x / \delta=0$

streamwise direction and flanked on either side in the spanwise direction by a region of negative correlation (marked by blue). This confirms the existence of VLSMs in the form of alternating low- and high-speed zones. Moreover, the correlated regions are inclined upward along the streamwise direction and more elongated towards the upstream direction, and the region of positive correlation extends vertically over a large part of the boundary layer (almost from the bottom to the top).

The instantaneous velocity fields shown in Fig. 3 provide evidence of large-scale roll modes accompanying large-scale elongated high-speed regions. To verify statistically whether the coexistence of large-scale roll modes is also an essential feature of VLSMs, we examine the conditionally-averaged flow field. The obtained velocity fields are normalized by the friction velocity as well. Figure 11 presents the conditionally-averaged flow field with 


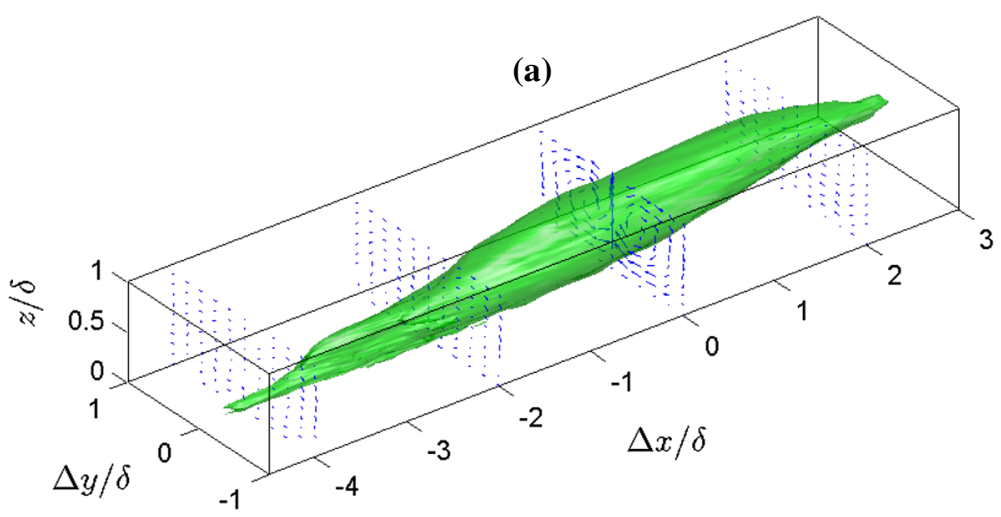

(b)

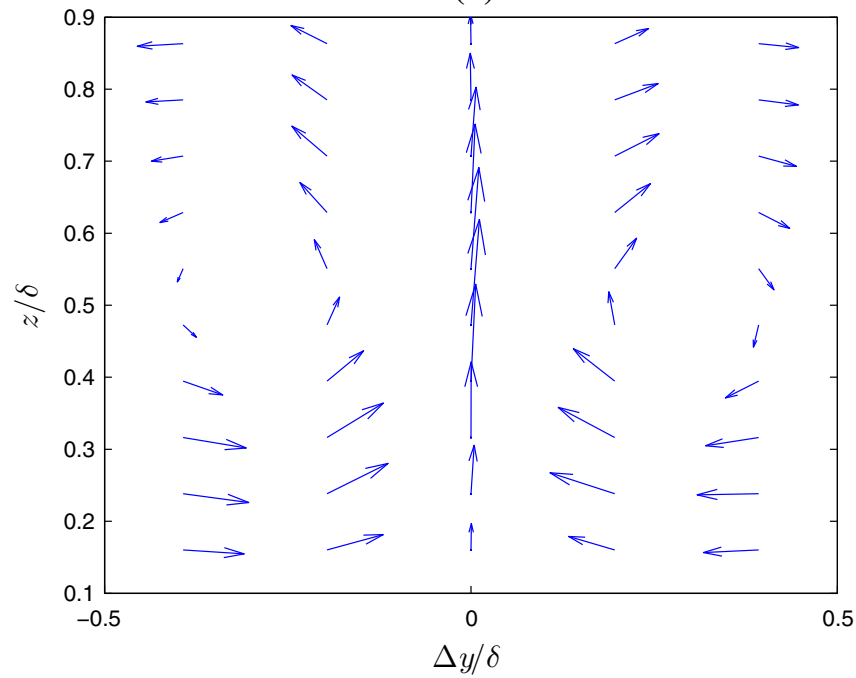

Fig. 12 Conditionally-averaged flow field. The conditional event is a low-speed event (of negative $u$ fluctuation) at $\Delta x=0, \Delta y=0, z=\delta / 2$. a Green iso-surfaces show negative $u$ fluctuation $(\leq-0.22)$; vector fields for the velocity components $v$ and $w$ are shown on spanwise/wall-normal planes at $\Delta x / \delta=-4,-2,0$, and 2. b Enlarged view of the plot of the vector field on the spanwise/wall-normal plane at $\Delta x / \delta=0$

the conditional event being a high-speed event at $\Delta x=0, \Delta y=0, z=\delta / 2$. In Fig. 11a, the high-speed region is shown by the iso-surface of positive $u$ fluctuation. Large-scale roll modes can be seen from the cross-plane plots displaying velocity vectors as arrows with components $(v, w)$ at the grid points. The combined plots in Fig. 11a and b depict clearly that the high-speed event is accompanied by a counter-rotating vortex pair with a downwash occurring inside the high-speed region. In a similar way, Fig. 12 presents the conditionallyaveraged flow field with the conditional event being a low-speed event at the same reference point. Again, it shows clearly that the low-speed event is accompanied by a counter-rotating vortex pair, but with an upwash occurring inside the low-speed region. These large-scale roll modes have been previously observed in laboratory experiments of turbulent boundary layers, through either a volumetric linear stochastic estimation of the conditionally-averaged velocity field (Marusic and Hutchins 2008) or a direct visualization of the instantaneous 
velocity field (Hutchins et al. 2005). In a recent study based on field experimental data of the neutral ABL (Hutchins et al. 2012), the authors applied the linear stochastic estimation method to show that "the low momentum fluid in the streamwise direction is accompanied by counter-rotating roll modes across the span of the flow." It is important to note that the field measurements were made below $30 \mathrm{~m}$ above the ground and the estimated ABL thickness was $60 \mathrm{~m}$, hence the observed large-scale modes are of a smaller scale than those found here. Nevertheless, similar structures found by Drobinski et al. (2004) using lidar data are of a comparable scale with those shown in Figs. 11 and 12 (see Fig. 11 of Drobinski et al. (2004), where the spanwise scale is around $1 \mathrm{~km}$ ). DNS studies (Toh and Itano 2005; Del Álamo et al. 2006) have also noted such vortex pairs associated with the large-scale structure. Del Álamo et al. (2006) have suggested that the vortex pair "...is statistically important, because it takes part in the stirring of the mean profile that leads to the generation of the large scales of $u$." In a recent DNS study of a turbulent boundary layer, Lee and Sung (2011) found that, due to these associated strong swirling motions, VLSMs contributed to approximately $45 \%$ of the total Reynolds shear stress.

\section{Conclusions}

We have focused on the nature of very-large-scale motions (VLSMs) in the ABL, and carried out LES at a very high friction Reynolds number, $R e_{\tau} \sim \mathcal{O}\left(10^{8}\right)$. The vertical height of the computational domain is $L_{z}=1000 \mathrm{~m}$, which corresponds to the thickness of the boundary layer. In order to properly capture VLSMs, which scale with the boundary-layer thickness, the horizontal dimensions of the simulation domain are chosen to be $L_{x}=32 \pi L_{z}$ and $L_{y}=4 \pi L_{z}$ respectively, which are much larger than the standard values of $L_{x}=L_{y}=$ $2 \pi L_{z}$, especially in the streamwise direction. It has been shown that the contributions to the resolved TKE and the resolved shear stress from streamwise wavelengths larger than $10 L_{z}$ are significant (up to 27 and $31 \%$ respectively). Hence, the very large domain size adopted here is necessary for the purpose of investigating VLSMs, while the use of the standard domain size could lead to unphysical effects due to the truncation of the important VLSMs.

Flow visualization and statistical analysis have been applied to characterize the spatially coherent structures associated with VLSMs. The instantaneous velocity fields in streamwise/spanwise planes show very long meandering zones of low-speed fluid with negative fluctuations of the streamwise velocity component, which look like the large-scale streaks observed in many atmospheric surface-layer flows. Each low-speed streak is flanked on either side by a high-speed streak, and vice versa. The pre-multiplied power spectra and two-point correlations indicate that the scales of these streak-like structures are very large, up to $20 \mathrm{~L}_{z}$ in the streamwise direction and $0.5 L_{z}$ in the spanwise direction, which are consistent with what have been termed VLSMs by Kim and Adrian (1999). These features are similar to those found in the logarithmic and outer regions of laboratory-scale boundary layers by DNS and experiments conducted at low to moderate Reynolds numbers. Both instantaneous and statistical results indicate that the size of these very-large-scale structures first increases with increasing distance from the wall, then remains nearly constant.

The three-dimensional correlation map and conditional average of the three components of velocity have been applied to explore the 3D structure of VLSMs. The results clearly show that the low-speed and high-speed regions possess the same elongated ellipsoid-like structure, which is inclined upward along the streamwise direction and extends almost from the bottom to the top of the boundary layer. Moreover, these structures are accompanied by counter-rotating roll modes in the cross-section perpendicular to the streamwise direction. 
These findings are in agreement with recent observations made from field campaigns in a neutral ABL (Hutchins et al. 2012).

It is important to note that the truly neutral ABL studied herein is an idealized case and, therefore, there are several important physical phenomena that require further study. First, Coriolis accelerations will produce a change of wind direction with height, which will influence the topology of VLSMs due to their extreme length. In addition, thermal stability will also affect the structure and evolution of VLSMs. Future research will focus on understanding those effects.

Acknowledgments This work was supported by a grant from the Swiss National Supercomputing Centre (CSCS) under project ID s495. The authors also would like to thank the anonymous reviewers for their valuable comments and suggestions to improve the quality of the paper.

\section{References}

Abe H, Kawamura H, Choi H (2004) Very large-scale structures and their effects on the wall shear-stress fluctuations in a turbulent channel flow up to $R e_{\tau}=640$. J Fluid Eng-T ASME 126(5):835-843

Abkar M, Porté-Agel F (2012) A new boundary condition for large-eddy simulation of boundary-layer flow over surface roughness transitions. J Turbul 13:N23

Adrian RJ (2007) Hairpin vortex organization in wall turbulence. Phys Fluids 19(4):041301

Albertson JD, Parlange MB (1999) Surface length scales and shear stress: Implications for land-atmosphere interaction over complex terrain. Water Resour Res 35(7):2121-2132

Anderson W, Passalacqua P, Porté-Agel F, Meneveau C (2012) Large-eddy simulation of atmospheric boundary-layer flow over fluvial-like landscapes using a dynamic roughness model. Boundary-Layer Meteorol 144(2):263-286

Andren A (1994) Large-eddy simulation of a neutrally stratified boundary layer: a comparison of four computer codes. Q J R Meteorol Soc 120(520):1457-1484

Ayotte KW, Sullivan PP, Andren A, Doney SC, Holtslag AAM, Large WG, McWilliams JC, Moeng C-H, Otte MJ, Tribbia JJ, Wyngaard JC (1996) An evaluation of neutral and convective planetary boundary-layer parameterizations relative to large eddy simulations. Boundary-Layer Meteorol 79(1-2):131-175

Balakumar BJ, Adrian RJ (2007) Large- and very-large-scale motions in channel and boundary-layer flows. Philos Trans R Soc A 365(1852):665-681

Basu S, Porté-Agel F (2006) Large-eddy simulation of stably stratified atmospheric boundary layer turbulence: a scale-dependent dynamic modeling approach. J Atmos Sci 63(8):2074-2091

Boppe RS, Neu WL (1995) Quasi-coherent structures in the marine atmospheric surface layer. J Geophys Res 100(C10):20635-20648

Bou-Zeid E, Meneveau C, Parlange MB (2004) Large-eddy simulation of neutral atmospheric boundary layer flow over heterogeneous surfaces: blending height and effective surface roughness. Water Resour Res 40:W02505

Bou-Zeid E, Meneveau C, Parlange MB (2005) A scale-dependent lagrangian dynamic model for large eddy simulation of complex turbulent flows. Phys Fluids 17(2):025105

Cancelli DM, Chamecki M, Dias NL (2014) A large-eddy simulation study of scalar dissimilarity in the convective atmospheric boundary layer. J Atmos Sci 71:3-15

Carlotti P (2002) Two-point properties of atmospheric turbulence very close to the ground: comparison of a high resolution LES with theoretical models. Boundary-Layer Meteorol 104(3):381-410

Chung D, McKeon BJ (2010) Large-eddy simulation of large-scale structures in long channel flow. J Fluid Mech 661:341-364

Coceal O, Dobre A, Thomas TG, Belcher SE (2007) Structure of turbulent flow over regular arrays of cubical roughness. J Fluid Mech 589:375-409

Del Álamo JC, Jiménez J (2009) Estimation of turbulent convection velocities and corrections to Taylor's approximation. J Fluid Mech 640:5-26

Del Álamo JC, Jiménez J, Zandonade P, Moser RD (2004) Scaling of the energy spectra of turbulent channels. J Fluid Mech 500:135-144

Del Álamo JC, Jiménez J, Zandonade P, Moser RD (2006) Self-similar vortex clusters in the turbulent logarithmic region. J Fluid Mech 561:329-358 
Dennis DJC, Nickels TB (2008) On the limitations of Taylor's hypothesis in constructing long structures in a turbulent boundary layer. J Fluid Mech 614:197-206

Dennis DJC, Nickels TB (2011) Experimental measurement of large-scale three-dimensional structures in a turbulent boundary layer, Part 2. Long structures. J Fluid Mech 673:218-244

Ding F, Palarya S, Lin YL (2001) Large-eddy simulations of the atmospheric boundary layer using a new subgrid-scale model: I. Slightly unstable and neutral cases. Environ Fluid Mech 1(1):29-47

Drobinski P, Carlotti P, Newsom RK, Banta RM, Foster RC, Redelsperger JL (2004) The structure of the near-neutral atmospheric surface layer. J Atmos Sci 61(6):699-714

Dubos T, Drobinski P, Carlotti P (2008) Turbulence anisotropy carried by streaks in the neutral atmospheric surface layer. J Atmos Sci 65(8):2631-2645

Finnigan JJ, Shaw RH, Patton EG (2009) Turbulence structure above a vegetation canopy. J Fluid Mech 637:387-424

Foster RC, Vianey F, Drobinski P, Carlotti P (2006) Near-surface coherent structures and the vertical momentum flux in a large-eddy simulation of the neutrally-stratified boundary layer. Boundary-Layer Meteorol 120(2):229-255

Ganapathisubramani B, Longmire EK, Marusic I (2003) Characteristics of vortex packets in turbulent boundary layers. J Fluid Mech 478:35-46

Ganapathisubramani B, Hutchins N, Monty JP, Chung D, Marusic I (2012) Amplitude and frequency modulation in wall turbulence. J Fluid Mech 712:61-91

Guala M, Hommema SE, Adrian RJ (2006) Large-scale and very-large-scale motions in turbulent pipe flow. J Fluid Mech 554:521-542

Guala M, Metzger M, McKeon BJ (2011) Interactions within the turbulent boundary layer at high Reynolds number. J Fluid Mech 666:573-604

Hambleton WT, Hutchins N, Marusic I (2006) Simultaneous orthogonal-plane particle image velocimetry measurements in a turbulent boundary layer. J Fluid Mech 560:53-64

Högström U, Hunt JCR, Smedman AS (2002) Theory and measurements for turbulence spectra and variances in the atmospheric neutral surface layer. Boundary-Layer Meteorol 103(1):101-124

Horiguchi M, Hayashi T, Hashiguchi H, Ito Y, Ueda H (2010) Observations of coherent turbulence structures in the near-neutral atmospheric boundary layer. Boundary-Layer Meteorol 136(1):25-44

Hoyas S, Jiménez J (2006) Scaling of the velocity fluctuations in turbulent channels up to $R e_{\tau}=2003$. Phys Fluids 18(1):011702

Hunt JCR, Carlotti P (2001) Statistical structure at the wall of the high Reynolds number turbulent boundary layer. Flow Turbul Combust 66(4):453-475

Hunt JCR, Morrison JF (2000) Eddy structure in turbulent boundary layers. Eur J Mech B 19(5):673-694

Hutchins N, Marusic I (2007a) Evidence of very long meandering features in the logarithmic region of turbulent boundary layers. J Fluid Mech 579:1-28

Hutchins N, Marusic I (2007b) Large-scale influences in near-wall turbulence. Philos Trans R Soc A 365(1852):647-664

Hutchins N, Hambleton WT, Marusic I (2005) Inclined cross-stream stereo particle image velocimetry measurements in turbulent boundary layers. J Fluid Mech 541:21-54

Hutchins N, Chauhan K, Marusic I, Monty J, Klewicki J (2012) Towards reconciling the large-scale structure of turbulent boundary layers in the atmosphere and laboratory. Boundary-Layer Meteorol 145(2):273-306

Inagaki A, Kanda M (2010) Organized structure of active turbulence over an array of cubes within the logarithmic layer of atmospheric flow. Boundary-Layer Meteorol 135(2):209-228

Khanna S, Brasseur JG (1998) Three-dimensional buoyancy- and shear-induced local structure of the atmospheric boundary layer. J Atmos Sci 55(5):710-743

Kim KC, Adrian RJ (1999) Very large-scale motion in the outer layer. Phys Fluids 11(2):417-422

Kirkil G, Mirocha J, Bou-Zeid E, Chow F, Kosović B (2012) Implementation and evaluation of dynamic subfilter-scale stress models for large-eddy simulation using WRF. Mon Weather Rev 140(1):266-284

Kleissl J, Kumar V, Meneveau C, Parlange MB (2006) Numerical study of dynamic Smagorinsky models in large-eddy simulation of the atmospheric boundary layer: Validation in stable and unstable conditions. Water Resour Res 42:W06D10

Kumar V, Kleissl J, Meneveau C, Parlange MB (2006) Large-eddy simulation of a diurnal cycle of the atmospheric boundary layer: atmospheric stability and scaling issues. Water Resour Res W06D42:09

Lee JH, Sung HJ (2011) Very-large-scale motions in a turbulent boundary layer. J Fluid Mech 673:80-120

Lee JH, Sung HJ (2013) Comparison of very-large-scale motions of turbulent pipe and boundary layer simulations. Phys Fluids 25(4):045103

Lin CL, McWilliams JC, Moeng CH, Sullivan PP (1996) Coherent structures and dynamics in a neutrally stratified planetary boundary layer flow. Phys Fluids 8(10):2626-2639 
Liu Z, Adrian RJ, Hanratty TJ (2001) Large-scale modes of turbulent channel flow: transport and structure. J Fluid Mech 448:53-80

Lu H, Porté-Agel F (2010) A modulated gradient model for large-eddy simulation: application to a neutral atmospheric boundary layer. Phys Fluids 22(1):1-12

Ludwig F, Chow F, Street R (2009) Effect of turbulence models and spatial resolution on resolved velocity structure and momentum fluxes in large-eddy simulations of neutral boundary layer flow. J Appl Meteorol Clim 48(6):1161-1180

Marusic I, Hutchins N (2008) Study of the log-layer structure in wall turbulence over a very large range of Reynolds number. Flow Turbul Combust 81(1-2):115-130

Mathis R, Hutchins N, Marusic I (2009) Large-scale amplitude modulation of the small-scale structures in turbulent boundary layers. J Fluid Mech 628:311-337

Mejia-Alvarez R, Wu Y, Christensen K (2014) Observations of meandering superstructures in the roughness sublayer of a turbulent boundary layer. Int J Heat Fluid Flow 48:43-51

Meneveau C, Lund T, Cabot W (1996) A Lagrangian dynamic subgrid-scale model of turbulence. J Fluid Mech 319:353-385

Mirocha J, Lundquist J, Kosović B (2010) Implementation of a nonlinear subfilter turbulence stress model for large-eddy simulation in the advanced research WRF model. Mon Weather Rev 138(11):4212-4228

Mirocha J, Kirkil G, Bou-Zeid E, Chow F, Kosović B (2013) Transition and equilibration of neutral atmospheric boundary layer flow in one-way nested large-eddy simulations using the weather research and forecasting model. Mon Weather Rev 141(3):918-940

Moeng CH, Sullivan PP (1994) A comparison of shear- and buoyancy-driven planetary boundary layer flows. J Atmos Sci 51(7):999-1022

Morrison JF (2007) The interaction between inner and outer regions of turbulent wall-bounded flow. Philos Trans R Soc A 365(1852):683-698

Newsom R, Calhoun R, Ligon D, Allwine J (2008) Linearly organized turbulence structures observed over a suburban area by dual-doppler lidar. Boundary-Layer Meteorol 127(1):111-130

Porté-Agel F (2004) A scale-dependent dynamic model for scalar transport in large-eddy simulations of the atmospheric boundary layer. Boundary-Layer Meteorol 112(1):81-105

Porté-Agel F, Meneveau C, Parlange MB (2000) A scale-dependent dynamic model for large-eddy simulation: application to a neutral atmospheric boundary layer. J Fluid Mech 415:261-284

Priymak VG, Miyazaki T (1994) Long-wave motions in turbulent shear flows. Phys Fluids 6(10):3454-3464

Shaw RH, Schumann U (1992) Large-eddy simulation of turbulent flow above and within a forest. BoundaryLayer Meteorol 61(1-2):47-64

Stoll R, Porté-Agel F (2006) Dynamic subgrid-scale models for momentum and scalar fluxes in large-eddy simulations of neutrally stratified atmospheric boundary layers over heterogeneous terrain. Water Resour Res 42(1):W01409

Stoll R, Porté-Agel F (2008) Large-eddy simulation of the stable atmospheric boundary layer using dynamic models with different averaging schemes. Boundary-Layer Meteorol 126(1):1-28

Tang ZQ, Jiang N, Schröder A, Geisler R (2012) Tomographic PIV investigation of coherent structures in a turbulent boundary layer flow. Acta Mech Sinica 28(3):572-582

Toh S, Itano T (2005) Interaction between a large-scale structure and near-wall structures in channel flow. J Fluid Mech 524:249-262

Tomkins CD, Adrian RJ (2003) Spanwise structure and scale growth in turbulent boundary layers. J Fluid Mech 490:37-74

Van der Hoven I (1957) Power spectrum of horizontal wind speed in the frequency range from 0.0007 to 900 cycles per hour. J Meteorol 14(2):160-164

Wilczak JM, Tillman JE (1980) The 3-dimensional structure of convection in the atmospheric surface layer. J Atmos Sci 37(11):2424-2443

Wu X, Baltzer JR, Adrian RJ (2012) Direct numerical simulation of a 30R long turbulent pipe flow at $R^{+}=685$ : large-and very large-scale motions. J Fluid Mech 698:235-281

Young GS, Kristovich DAR, Hjelmfelt MR, Foster RC (2002) Rolls, streets, waves, and more: a review of quasi-two-dimensional structures in the atmospheric boundary layer. Bull Am Meteorol Soc 83(7):9971001 